\title{
Apparent and Intrinsic Evolution of Active Region Upflows
}

\author{
Deborah Baker $^{1}\left(\mathbb{D} \cdot\right.$ Miho Janvier $^{2}$ (D) \\ Pascal Démoulin $^{3}$ (D) Cristina H. Mandrini ${ }^{4,5}$
}

Received: 24 November 2016 / Accepted: 23 February 2017 / Published online: 22 March 2017

(C) The Author(s) 2017. This article is published with open access at Springerlink.com

\begin{abstract}
We analyze the evolution of Fe XII coronal plasma upflows from the edges of ten active regions (ARs) as they cross the solar disk using the Hinode Extreme Ultraviolet Imaging Spectrometer (EIS) to do this. Confirming the results of Démoulin et al. (Sol. Phys. 283, 341, 2013), we find that for each AR there is an observed long-term evolution of the upflows. This evolution is largely due to the solar rotation that progressively changes the viewpoint of dominantly stationary upflows. From this projection effect, we estimate the unprojected upflow velocity and its inclination to the local vertical. AR upflows typically fan away from the AR core by $40^{\circ}$ to nearly vertical for the following polarity. The span of inclination angles is more spread out for the leading polarity, with flows angled from $-29^{\circ}$ (inclined toward the AR center) to $28^{\circ}$ (directed away from the AR). In addition to the limb-to-limb apparent evolution, we identify an intrinsic evolution of the upflows that is due to coronal activity, which is AR dependent. Furthermore, line widths are correlated with
\end{abstract}

Electronic supplementary material The online version of this article

(doi:10.1007/s11207-017-1072-9) contains supplementary material, which is available to authorized users.

\footnotetext{
D. Baker

deborah.baker@ucl.ac.uk

M. Janvier

mjanvier@ias.u-psud.fr

P. Démoulin

Pascal.Demoulin@obspm.fr

C.H. Mandrini

mandrini@iafe.uba.ar

1 Mullard Space Science Laboratory, University College London, Holmbury, St. Mary, Dorking, Surrey, KT22 9XF, UK

2 Institut d'Astrophysique Spatiale, CNRS, Univ. Paris-Sud, Université Paris-Saclay, Bât. 121, 91405 Orsay cedex, France

3 Observatoire de Paris, LESIA, UMR 8109 (CNRS), 92195 Meudon Principal cedex, France

4 Instituto de Astronomía y Física del Espacio (IAFE), UBA-CONICET, Buenos Aires, Argentina

5 Facultad de Ciencias Exactas y Naturales (FCEN), UBA, Buenos Aires, Argentina
} 
Doppler velocities only for the few ARs with the highest velocities. We conclude that for the line widths to be affected by the solar rotation, the spatial gradient of the upflow velocities must be large enough such that the line broadening exceeds the thermal line width of Fe XII. Finally, we find that upflows occurring in pairs or multiple pairs are a common feature of ARs observed by Hinode/EIS, with up to four pairs present in AR 11575. This is important for constraining the upflow-driving mechanism as it implies that the mechanism is not local and does not occur over a single polarity. AR upflows originating from reconnection along quasi-separatrix layers between overpressure AR loops and neighboring underpressure loops is consistent with upflows occurring in pairs, unlike other proposed mechanisms that act locally in one polarity.

Keywords Active regions, velocity field · Active regions, magnetic fields

\section{Introduction}

\subsection{General Characteristics of Active Region Upflows}

Since the launch of the Hinode Extreme Ultraviolet Imaging Spectrometer (EIS), steady coronal plasma upflows have been observed to emanate from the edges of active regions (ARs; Sakao et al., 2007; Harra et al., 2008). Their blueshifted velocities are in the range $[5,50] \mathrm{km} \mathrm{s}^{-1}$ for the Fe XII $195 \AA$ emission line. Similar velocities have been measured at much higher spatial resolution by the Interface Region Imaging Spectrograph (IRIS), also using Fe XII (Testa, De Pontieu, and Hansteen, 2016). Some ARs show large bluewing asymmetries exceeding $100 \mathrm{~km} \mathrm{~s}^{-1}$ (Bryans, Young, and Doschek, 2010; De Pontieu and McIntosh, 2010; Tian et al., 2011; Brooks and Warren, 2012; Vanninathan et al., 2015). Upflow temperatures range from 1-2.5 MK (Warren et al., 2011) and the Doppler velocities increase with temperature in the corona (Del Zanna, 2008).

Typically, these large-scale upflows are located above monopolar regions of strong magnetic field (Doschek et al., 2008; Del Zanna, 2008). Upflowing plasma is observed by Hinode/EIS in approximately the same locations for at least 8-9 days as ARs transit the solar disk (Démoulin et al., 2013). Recently, Zangrilli and Poletto (2016) have shown that the upflows persist for much longer periods. Using data from Solar and Heliospheric $\mathrm{Ob}$ servatory's Ultraviolet Coronagraph Spectrometer (SOHO/UVCS), they demonstrated that upflows from AR 8100 extended into the intermediate corona to become outflows and persisted for the entire lifetime of the AR, which spanned five solar rotations.

\subsection{Upflow-Driving Mechanisms}

Baker et al. (2009), van Driel-Gesztelyi et al. (2012), Démoulin et al. (2013), and Mandrini et al. (2015) have demonstrated that upflows occur along quasi-separatrix layers (QSLs), which are thin 3D volumes where magnetic field lines display strong gradients in magnetic connectivity (Démoulin et al., 1996). QSLs are preferential locations for current sheet development and magnetic reconnection (e.g. Aulanier et al., 2006; see Janvier, 2017 for a review). QSLs are typically present between the core of the AR and the surrounding regions (including open field). The AR core has overpressure loops compared to the loops in these nearby regions. As the AR is developing, its core grows so that associated magnetic field motions can build current layers at the QSLs. This process is expected to drive a long-term 
reconnection process (ranging from quasi-continuous to a series of frequent small reconnection events). After reconnection, the resulting pressure gradient drives the upflows along the reconnected field lines.

A similar mechanism was proposed by Del Zanna et al. (2011), whereby upflows were related to a coronal null-point of a pseudostreamer. Interchange reconnection (i.e. taking place between AR loops and open field lines) can take place at the coronal null, creating a steep pressure gradient and a rarefaction wave in the reconnected loops (Bradshaw, Aulanier, and Del Zanna, 2011). As QSLs are the generalized form of separatrices and null points in $3 \mathrm{D}$, and because they include them, reconnection at these topological locations is a subset of QSL reconnection. Clearly, magnetic reconnection is likely to play a key role in driving AR plasma upflows.

Several other mechanisms have been proposed as direct or indirect drivers of AR upflows observed by Hinode/EIS, such as AR expansion (Murray et al., 2010), waves (e.g. Wang et al., 2009; Verwichte et al., 2010; Ofman, Wang, and Davila, 2012; Galsgaard et al., 2015), coronal plasma circulation (Marsch et al., 2008), chromospheric evaporation (Del Zanna, 2008), and type II spicules (e.g. De Pontieu et al., 2009; De Pontieu and McIntosh, 2010; Tian et al., 2012), among others.

\subsection{Structure of This Article}

Démoulin et al. (2013) used Hinode/EIS data to derive the physical properties of the largescale upflows observed on both sides of AR 10978 as it crossed the solar disk. A leastsquares fitting of a stationary flow model to the data provided the means to distinguish apparent evolution that is due to line-of-sight (LOS) projection effects from intrinsic flow evolution. It was shown that the upflows have a strong collimated stationary component.

In this study we apply the same analysis as in Démoulin et al. (2013) to nine additional ARs with good limb-to-limb EIS coverage. Section 2 provides a summary of the steady-flow model and key results from AR 10978. In Section 3 we summarize the EIS observations in our AR sample. We analyze the limb-to-limb Fe XII upflow evolution for three case studies in Section 4. Then we generalize the results for all ARs before studying the evolution of line widths in Section 5. We compare the inclinations of the stationary flows of AR 10926 with those of the coronal magnetic field using a linear force-free field extrapolation of the AR photospheric LOS field (Section 6). In Section 7 we show that upflows in AR 11575 occur in four pairs, which enables us to investigate any similarity between the two polarities of the same pair. Finally, we summarize our results and draw our conclusions (Section 8).

\section{Steady-Flow Model Applied to AR 10978}

\subsection{Steady-Flow Model}

Démoulin et al. (2013) analyzed the limb-to-limb evolution of plasma upflows from AR 10978 using Hinode/EIS. A global evolution of the Doppler velocities of the AR upflow is apparent as it crosses the solar disk from 6-16 December 2007. On the eastern side of the AR, upflow velocities increase almost continuously with time before reversing early on 15 December. A similar evolution takes place on the western side, but the change from increasing to decreasing upflow velocities occurs four days earlier. Such a coherent, largescale evolution is clear evidence of a LOS projection effect evolving with the AR position on the disk, thereby demonstrating that the upflows have a strongly collimated stationary 
component. Upflow velocities on either side of the AR peak when the stationary component is parallel to the LOS of Hinode/EIS.

A stationary-flow model was developed to quantify the effect of the evolving LOS projection in order to separate apparent velocity evolution that is due to solar rotation from intrinsic velocity evolution that is due to some form of activity such as flux emergence, flares, and coronal mass ejections (CMEs). In applying this simple model to EIS Doppler velocity data of AR 10978, it was assumed that the plasma upflows are continuously driven with the same velocity magnitude and orientation in the local solar frame. The main equations of the model are presented here for convenience. See Démoulin et al. (2013) for a complete account of the model and its underlying assumptions, uncertainties, and limitations.

In spherical coordinates with the radial direction $[r]$, the longitude $[\varphi]$, and the latitude $[\theta]$, the velocity $[\boldsymbol{V}]$ is

$$
\boldsymbol{V}=V_{r} \hat{\mathbf{u}}_{r}+V_{\varphi} \hat{\mathbf{u}}_{\varphi}+V_{\theta} \hat{\mathbf{u}}_{\theta},
$$

where $\hat{\mathbf{u}}_{r}, \hat{\mathbf{u}}_{\varphi}$, and $\hat{\mathbf{u}}_{\theta}$ are unit vectors. The position of the flow on the solar disk, normalized to the solar radius, is given by $X=\sin \varphi \cos \theta$ in the east-west direction and $Y=\sin \theta$ in the north-south direction. The observed velocity component, $V_{\|}$, is

$$
V_{\|}=V_{r}^{\prime} \sqrt{1-X^{2}-Y^{2}}-V_{\varphi} \frac{X}{1-Y^{2}} \quad \text { with } V_{r}^{\prime}=V_{r}-V_{\theta} \frac{Y}{\sqrt{1-Y^{2}}} .
$$

Intrinsic or temporal evolution of the stationary flows is indicated by deviations of the data from the fit to Equation (2).

\subsection{Key Results - AR 10978}

The inclination and angular spread of the strongest upflows in three coronal lines, Si VII, Fe XII, and Fe XV ( $\log T \approx 5.8,6.2$, and 6.3, respectively) are determined using the flow model for AR 10978. The deduced 3D geometry of the flows is consistent with that of a thin fan-like structure, in agreement with a magnetic field extrapolation of AR 10978. Fans on either side of the AR are tilted away from its core. A coherent fan structure is found on the eastern side (following polarity) of the AR, where the strongest flows are stationary, unlike on the western side (leading polarity), where in addition to a stationary component, a clear intrinsic evolution is related to new flux emergence on the sides of this polarity (Mandrini et al., 2015).

A dependence of the line widths that is similar to that of Doppler velocities with respect to the position of AR 10978 on the solar disk was previously found (Doschek et al., 2008; Bryans, Young, and Doschek, 2010). The contribution to the line width, $\left[W_{0}\right]$, which is independent of the AR's position, is very small, implying that the line width is primarily due to a large dispersion of velocities in the direction of the stationary flows (see Section 5 of this article and Section 4.4 of Démoulin et al. (2013) for a full explanation of $W_{0}$ ). Taken in their entirety, these results indicate that in the global picture the same upflows are detected in a number of spectral lines at coronal temperatures. Blueshifted plasma flows away from the AR core along magnetic field lines within a narrow angular range, but with a broad velocity range. The flows form a fan-like structure on either side of the AR as the collimated upflows expand with increasing height within the corona (see Figure 14 of Démoulin et al., 2013). The upflow driver(s) is (are) acting for extended periods, at least as long as the time it takes for the AR to cross the solar disk. The location of the flows within the magnetic configuration coupled with their narrow angular extent are compatible with a flow resulting from reconnection at QSLs, including separatrices (Baker et al., 2009; Del Zanna et al., 2011; van Driel-Gesztelyi et al., 2012; Démoulin et al., 2013; Mandrini et al., 2015). 


\section{Data and Analysis Method}

\subsection{Observations}

Nine new ARs are considered in this study, along with the results previously found for AR 10978. Observations are from the EIS instrument onboard Hinode (Culhane et al., 2007). ARs are selected from the EIS catalog based on how well their upflow regions are observed from limb to limb. In six cases, following polarities (FPs) and leading polarities (LPs) of the ARs are covered, but for four ARs only one polarity is within the EIS FOV throughout the disk transit time.

All of the ARs, with the exception of AR 11575, have a relatively simple bipolar magnetic structure and flux distribution, which is consistent with the structure and distribution of ARs in the decay phase of their lifetimes. LPs range from highly concentrated spots to dispersed magnetic field regions, while FPs consist only of dispersed field, with the exception of AR 10978, which contains coherent spots in both. Nine of the ten ARs are classified as large, with a total unsigned flux greater than $5 \times 10^{21} \mathrm{Mx}$ (van Driel-Gesztelyi and Green, 2015).

NOAA number, solar object locator (SOL) at the date and time of central meridian passage (CMP), and estimated minimum AR age are given in Table 1. It is not possible to directly determine the age of any of the ARs since none were observed during flux emergence. Instead, we provide a minimum age of the ARs solely based on the first detection at the solar east limb or on the far side using observations of the Solar Terrestrial Relations Observatory (STEREO) A and B spacecraft. Minimum ages range from 7 to 23 days. The description of the magnetic flux distribution for LPs and FPs and the total AR unsigned flux at CMP are given in Table 2. Information and results pertaining to AR 10978 from Démoulin et al. (2013) are included in the tables of this article for comparison purposes.

\subsection{Data Reduction}

The rastered images are constructed with the 1" and 2" slits in scanning mode. A total of 24 different EIS studies with varying exposure times, fields of view (FOV), and spectral line lists are used in this investigation. See Table 4 of the Appendix for EIS study details including study number, FOV, slit size, exposure time, and total raster time. Data reduction is performed with standard SolarSoft EIS routines, which correct for dark current, hot, warm,

Table 1 ARs included in the limb-to-limb upflow study. The minimum age is given for the first observation of the ARs at the east limb or on the far side.

\begin{tabular}{llc}
\hline $\begin{array}{l}\text { NOAA } \\
\text { AR }\end{array}$ & $\begin{array}{l}\text { Solar object locator } \\
\text { (SOL) at CMP }\end{array}$ & $\begin{array}{l}\text { Min. age } \\
\text { (days) }\end{array}$ \\
\hline 10926 & 2006-12-01T16:00:00L138C097 & 8 \\
10938 & 2007-01-18T16:00:00L226C086 & 8 \\
10942 & 2007-02-22T08:00:00L130C102 & 8 \\
10953 & 2007-05-01T17:00:00L308C100 & 7 \\
10961 & $2007-07-01 T 19: 00: 00 L 220 C 109$ & 7 \\
10978 & $2007-12-11 T 22: 00: 00 L 226 C 102$ & 9 \\
11389 & $2012-01-03 T 17: 00: 00 L 082 C 111$ & 21 \\
11564 & $2012-09-05 T 21: 00: 00 L 071 C 104$ & 23 \\
11575 & $2012-09-24 T 21: 00: 00 L 181 C 084$ & 22 \\
11589 & $2012-10-15 T 17: 00: 00 L 266 C 079$ & 21 \\
\hline
\end{tabular}


Table 2 Global properties of ARs included in the limb-to-limb upflow study.

\begin{tabular}{llll}
\hline $\begin{array}{l}\text { NOAA } \\
\text { AR }\end{array}$ & $\begin{array}{l}\text { Following } \\
\text { polarity }\end{array}$ & $\begin{array}{l}\text { Leading } \\
\text { polarity }\end{array}$ & $\begin{array}{l}\text { Tot. unsigned flux } \\
\text { at CMP (Mx) }\end{array}$ \\
\hline 10926 & Dispersed & Strong spots + dispersed & $7.4 \times 10^{21}$ \\
10938 & Dispersed & Dispersed & $9.8 \times 10^{21}$ \\
10942 & Dispersed & Dispersed & $3.5 \times 10^{21}$ \\
10953 & Dispersed & Strong spot & $1.6 \times 10^{22}$ \\
10961 & Dispersed & Strong spots & $5.9 \times 10^{21}$ \\
10978 & Spots + dispersed & Spots + dispersed & $1.9 \times 10^{22}$ \\
11389 & Dispersed & Strong spot + dispersed & $1.5 \times 10^{22}$ \\
11564 & Dispersed & Dispersed & $1.5 \times 10^{22}$ \\
11575 & Dispersed & Strong spot & $1.0 \times 10^{22}$ \\
11589 & Dispersed & Dispersed & $1.4 \times 10^{22}$ \\
\hline
\end{tabular}

and dusty pixels, detector bias, and cosmic rays. Further corrections are made for slit tilt and spectrum drift that is due to the temperature variation throughout the spacecraft orbit.

Coronal spectral line profiles have been shown to have an extended blue wing in the upflow regions for a few ARs, e.g. AR 10978 in Bryans, Young, and Doschek (2010) and Brooks and Warren (2012), AR 10938 in Hara et al. (2008) and Peter (2010), both of which are included in our AR study, and AR 11123 in Vanninathan et al. (2015). All of these authors required a second velocity component to fit the asymmetric Fe XII - XV profiles as they found significant deviations from single Gaussian fits. In most cases, a double Gaussian fit was sufficient to distinguish the primary and secondary velocity components of the AR upflows. Typical primary component velocities were $5-20 \mathrm{~km} \mathrm{~s}^{-1}$, whereas the secondary component velocities exceeded $100 \mathrm{~km} \mathrm{~s}^{-1}$ in all cases. Enhanced blue-wing velocities have been linked to, among other phenomena, jets (e.g. Vanninathan et al., 2015) and type II spicules (e.g. McIntosh and De Pontieu, 2009; Peter, 2010). In Démoulin et al. (2013), the results of double Gaussian fittings of the faster flows of AR 10978 could be trusted for a very limited number of pixels where upflow velocities were high enough to separate the secondary and primary components and where the emission measure was large enough to provide a reliable secondary component. For the other ARs in our study the variety of exposure times in the range $5-70$ s proved to be problematic for consistently separating the components for all data sets.

In addition to enhanced blue wings, the Fe XII $195.12 \AA$ line is blended with Fe XII at 195.18 А. The self-blend must be taken into account in regions of high density $>10^{10} \mathrm{~cm}^{-3}$, e.g. in AR cores (Young et al., 2009); however, in upflow regions densities are typically $\lesssim 10^{9} \mathrm{~cm}^{-3}$ (Bryans, Young, and Doschek, 2010; Culhane et al., 2014) so that we can ignore the effects of this blend. Hence, in the study presented here, single Gaussian functions are fitted to the Fe XII spectra in order to determine the total line intensity, the line width (full width at half maximum, FWHM), and the centroid wavelength.

\subsection{Deriving Steady Flows}

We apply the steady-flow model summarized in Section 2.1 to upflow streams associated with the LPs and/or FPs of all ARs in our study. When the flows are stationary and well collimated, the observed velocity from a given location within the AR is described by the model (Equation (2)). However, upflows have an unknown 3D geometry in the corona, and 
Figure 1 Diagram to illustrate the sign and the typical range of the inclination angle $[\delta]$ of the upflows with respect to the vertical for the leading (LP) and following polarities (FP) of an AR. The arrows show possible directions of the mean upflow velocity $[\boldsymbol{V}]$ in each polarity.

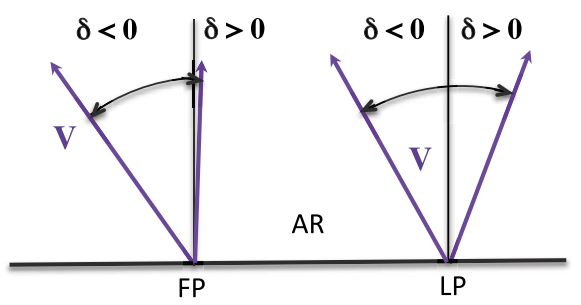

therefore it is not possible to follow an upflow coming from a fixed AR location (within the local solar frame). Instead, we proceed statistically over a full upflow region. When this region is defined by a given isocontour of $V_{\|}$, its extent is modified by the projection along the LOS (the selected region would be larger as the velocity is higher along the LOS). As a collimated upflow region has a relatively small extension in latitude and longitude and therefore $V_{\|}$is affected by a similar projection factor, the region(s) with the strongest flows is (are) essentially independent of the projection. In practice, only a fixed number of pixels with the strongest upflows are retained. This does not fully separate the effect of the variable extension from the evolving projection, but it is the most satisfactory method we have found (see Section 2.4 of Démoulin et al., 2013). We experimented with the number of data points from each raster observation in the range 20-100. We find that the effect on the derived mean velocities, line widths, and inclination angles is weak, which is consistent with the findings of Démoulin et al. (2013).

The classical convention is that $V_{\|}$is positive for flows directed away from the observer, therefore upflows have $V_{\|}<0$. For convenience, plots show $-V_{\|}$, i.e. positive values. The highest 50 values are selected in each data set, and the least-squares fitting to Equation (2) is applied to these data points. Mean velocity $[V]$, mean line width $[W]$, and mean inclination angle $[\delta]$ are determined from the fits for all available upflow regions. The angle $\delta$ measures the east-west inclination of the flows to the local vertical with the same sign convention in the FP and LP (Figure 1). The notations are the same as in Démoulin et al. (2013), where more explanations can be found.

\section{Steady-Flow Model Results}

Examples of the fitting to the steady-flow model for selected ARs are displayed in Figure 2. In the left panels, $-V_{\|} v s$. the $X$ position is plotted for four cases. Blue dots indicate the 50 highest $-V_{\|}$values selected in each data set, and red dots are their mean values. Black lines represent the least-squares fittings of Equation (2) to the data points. Fit results are given at the top of each panel for these ARs and are also provided for all ARs in Table 3.

Fluctuations of the velocities around the fitted values are displayed graphically in the right panels. Data points (blue dots) were corrected for projection effects using the fitted curves in the corresponding panels on the left. Red lines represent the mean values of the fit differences, and the black lines are \pm one standard deviation. This gives a straightforward view on the fluctuation level and provides a direct way to identify intrinsic activity (see for example the red arrow in Figure $2 \mathrm{~h}$ ). At the top of the plots we indicate two measures of the variation of the data values: the standard deviation $\left[\sigma_{t}\right]$ of all mean values (shown in red in the panels), and the mean of the standard deviation $\left[\left\langle\sigma_{\mathrm{obs}}\right\rangle\right]$ for each data set. They measure the fluctuations of the mean velocities with time and the mean value of the spatial fluctuations, respectively (for the highest velocities). 

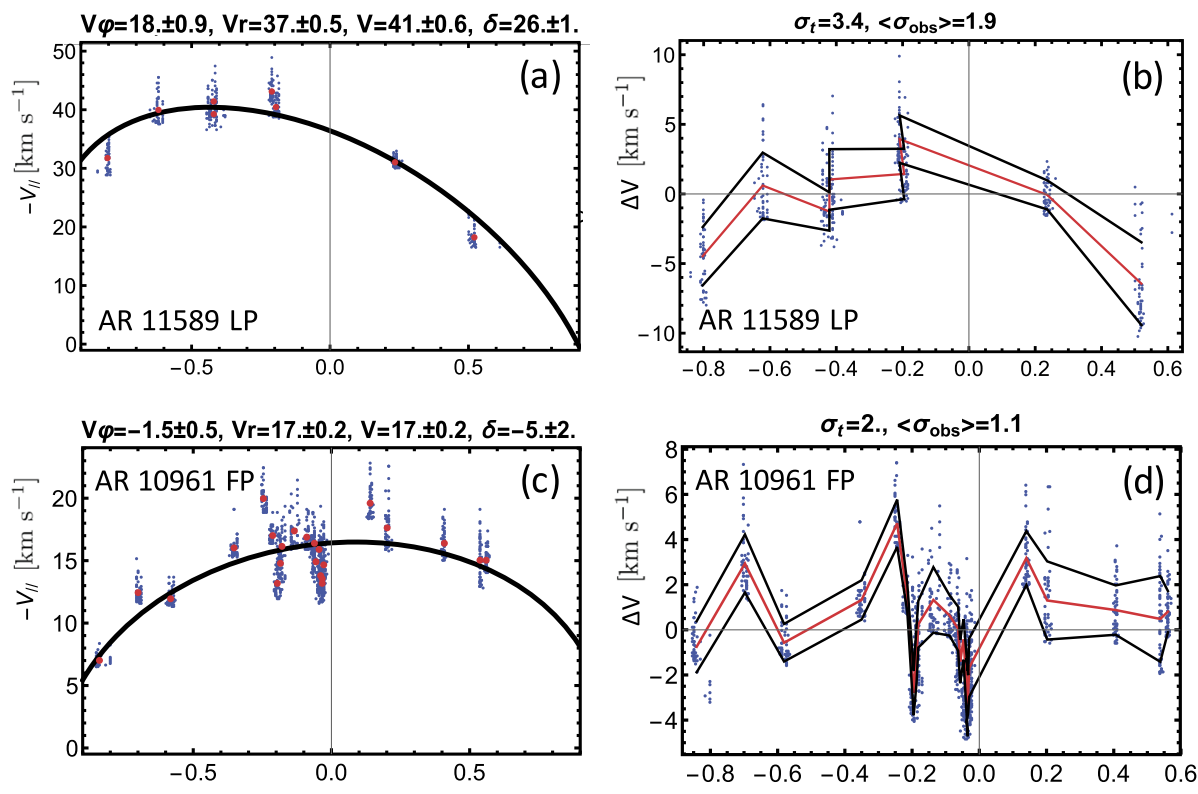

$\mathrm{V} \varphi=-6 . \pm 0.4, \mathrm{Vr}=9.4 \pm 0.1, \mathrm{~V}=11 . \pm 0.3, \delta=-33 . \pm 2$.
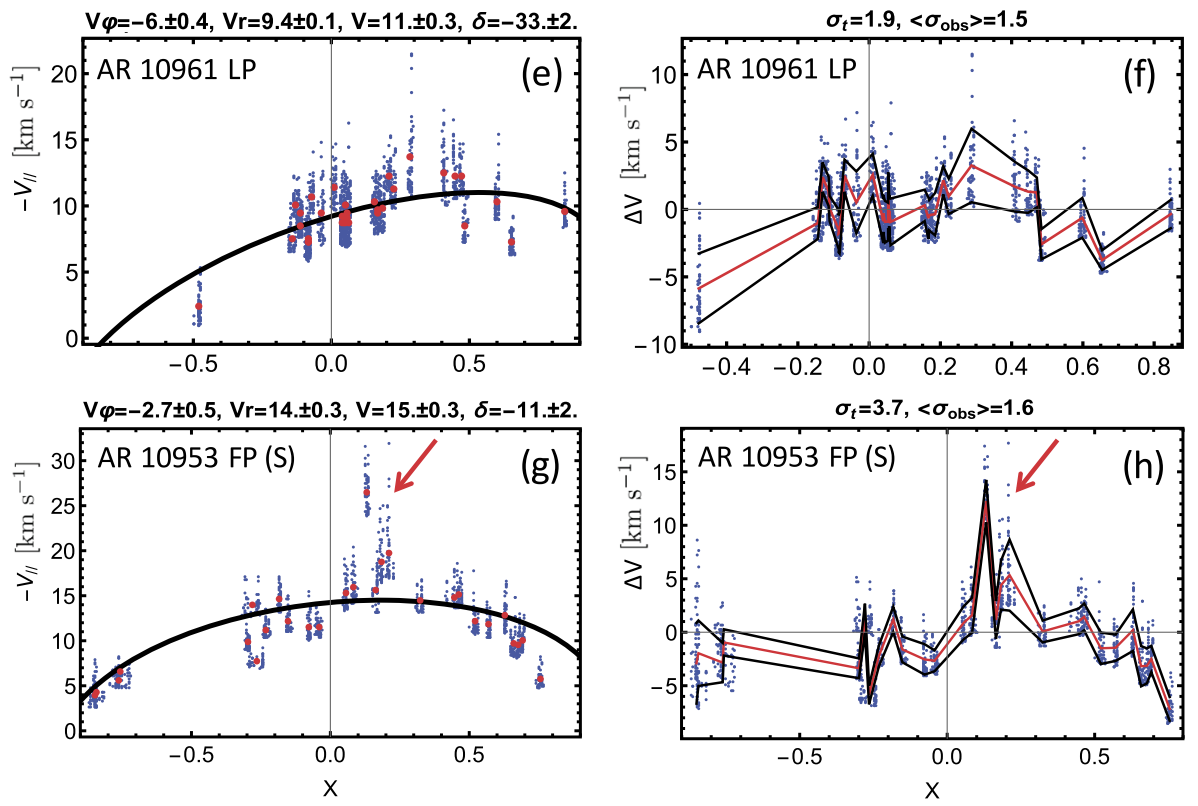

Figure 2 Left panels (top to bottom): Steady-flow model results for ARs 11589, 10961 (FP and LP), and $10953 \mathrm{~S}$ (southern bipole) showing the dependence of the LOS upflow velocity $\left[V_{\|}\right]$from the eastern to the western AR position normalized to the solar radius $[X]$. Blue dots indicate the 50 strongest $-V_{\|}$values selected in each data set, and red dots are mean values for each data set. The black line is the least-squares fitting of Equation (2) to these data points (in blue). Fit results are given at the top of each panel. Right panels: Fluctuations of the velocities around the fitted values corrected for projection effects. The red lines show the mean values of the fit difference, and the black lines correspond to \pm one standard deviation. $\sigma_{t}$ is the standard deviation of all mean values (red), and $\left\langle\sigma_{\mathrm{obs}}\right\rangle$ is the mean of the standard deviation for each data set. The red arrows point to the location of AR 10953 when the eruptions occurred (see Section 4.2). $V$ and $\delta$ at the top of panel $(\mathrm{g})$ are slightly different than in Table 3 because the fits include the activity times. 
Table 3 Results of the steady-flow model for ten ARs, including AR 10978. See Figure 1 for the sign convention of $\delta$. The values indicate the results of the fits where the intrinsic activity has been removed, so as to only keep the apparent evolution parameters of the ARs. NA in the different columns stands for unavailable data. Non-physical values are also found for the line broadening, meaning that the fit did not work in these cases. See Section 5 for an explanation of $W_{0}, W_{r}$, and $W_{\varphi}$. For AR $10978 \mathrm{FP}, W_{0}$ is abnormally small, therefore we only report "large".

\begin{tabular}{|c|c|c|c|c|c|c|}
\hline \multirow[t]{2}{*}{ NOAA AR } & \multicolumn{2}{|c|}{ Velocity $\left(\mathrm{km} \mathrm{s}^{-1}\right)$} & \multicolumn{2}{|c|}{$\left(W_{r}^{2}+W_{\varphi}^{2}\right)^{1 / 2} / W_{0}$} & \multicolumn{2}{|c|}{ Inclination $\delta$ (deg.) } \\
\hline & FP & LP & FP & LP & FP & LP \\
\hline 10926 & 18 & 14 & 0.29 & 0.45 & -40 & -12 \\
\hline 10938 & 17 & NA & 0.84 & NA & -4 & NA \\
\hline 10942 & 14 & NA & 0.34 & NA & 4 & NA \\
\hline $10953(\mathrm{~N})$ & 17 & 19 & 1 & 0.55 & -8 & 26 \\
\hline $10953(\mathrm{~S})$ & 14 & NA & 0.56 & $\mathrm{NA}$ & -10 & NA \\
\hline 10961 & 17 & 11 & 0.37 & 1.39 & -5 & -29 \\
\hline 10961 & NA & 9 & NA & 0.69 & NA & 21 \\
\hline 10978 & 32 & 34 & large & 3.39 & -39 & 19 \\
\hline 11389 & 18 & NA & 1.53 & NA & -10 & NA \\
\hline 11564 & 31 & 30 & 0.55 & 1.96 & -29 & 28 \\
\hline $11575(\mathrm{~N})$ & 12 & 12 & non-physical & 0.43 & -19 & -1 \\
\hline $11575(\mathrm{C})$ & 12 & 16 & 0.18 & 0.46 & -27 & 9 \\
\hline $11575(\mathrm{~S})$ & 11 & 20 & 0.85 & 2.29 & 2 & 11 \\
\hline 11589 & NA & 41 & NA & 0.32 & NA & 26 \\
\hline
\end{tabular}

In the following subsections, we use the least-squares fitting of the stationary flow model to distinguish apparent from intrinsic flow evolution. Deviations of the data from the model indicate the temporal evolution of the flows. We give a brief account of the results for two ARs that have high and low values of the derived velocities, respectively, but both display a dominant apparent evolution. Then we examine an AR in more detail in which an eruption occurred during the disk transit.

\subsection{Apparent Evolution - AR 11589 and AR 10961}

Hinode/EIS observed the western upflow region of AR 11589 from 10-17 October 2012. Throughout this period, the dispersing magnetic flux of both polarities spreads over an everincreasing area. The decaying AR has a bipolar magnetic configuration. Loops originating in the upflow region over the positive polarity (FP) connect externally with the negative polarity of the nearby AR 11592 to the northeast and the surrounding quiet Sun. The upflow region on the negative polarity (LP) extends into the negative field of a coronal hole immediately to the west. Activity is steady with $23 \mathrm{C}$-class flares attributed to the AR; however, no significant internal flux emergence or CMEs are detected.

The steady-flow model fit and the deviation from the fitting are shown in Figures 2a and $2 b$, respectively. From the curvature of the black line in the left plot, it is clear that the limb-to-limb evolution of upflows of the LP is dominated by the effects of the velocities projected onto the LOS. The derived mean velocity is $41 \mathrm{~km} \mathrm{~s}^{-1}$, which is the strongest in our study. The error of this parameter, derived from the fitted parameters, is estimated to be $\pm 0.6 \mathrm{~km} \mathrm{~s}^{-1}$. Overall, the model fit is very stable as the mean values of the fit differences (red line in Figure $2 \mathrm{~b}$ ) are $\lesssim 5 \mathrm{~km} \mathrm{~s}^{-1}$ until the AR approaches the western limb, where no 
data beyond approximately the +0.5 normalized AR position are available. The temporal dispersion $\left[\sigma_{t}\right]$ is $3.4 \mathrm{~km} \mathrm{~s}^{-1}$ and the spatial mean dispersion $\left[\left\langle\sigma_{\mathrm{obs}}\right\rangle\right]$ is $1.9 \mathrm{~km} \mathrm{~s}^{-1}$, which is a factor 10 and 20 times smaller than the mean velocity.

The two polarities of AR 10961 were observed by Hinode/EIS from 27 June to 6 July 2007. For most of the transit time, the isolated AR magnetic configuration is bipolar, although unlike AR 11589, its LP contains a strong coherent spot. Its total unsigned flux is $5.9 \times 10^{21} \mathrm{Mx}$. Activity is limited to a few jets and B-class flares in close proximity to the upflow region on the LP. In Figure 2, middle panels, the upflow streams on both sides of the AR exhibit a moderately strong dependence of $V_{\|}$on the normalized $X$-position of the AR. The mean velocity for the FP is $17.0 \pm 0.2 \mathrm{~km} \mathrm{~s}^{-1}$ and $11.0 \pm 0.3 \mathrm{~km} \mathrm{~s}^{-1}$ for the LP. The model fits are very good on either side of the AR. Values of $\sigma_{t}$ and $\left\langle\sigma_{\text {obs }}\right\rangle$ are similar for each polarity and much lower than those for AR 11589, while the values relative to the mean velocity are comparable.

In summary, AR 11589 and AR 10961 upflows exhibit a strongly collimated stationary component as they cross the solar disk, so that the observed evolution is dominantly due to projection. This is the case for both low (AR 10961) and high (AR 11589) $-V_{\|}$values. In our data sample, this result is the same for the other ARs that exhibit low activity levels.

\subsection{Intrinsic Evolution - AR 10953}

AR 10953 crossed the solar disk from 25 April to 6 May 2007. The AR had a simple bipolar configuration with a concentrated leading sunspot and dispersed FP. No significant flux emergence was observed. Flaring activity was limited to two C-class flares; however, there were several filament eruptions. Two of them occurred at 18:30 UT and 23:12 UT on 2 May (Okamoto et al., 2008; Su et al., 2009). Figure 3 shows STEREO B $195 \AA$ Antensity and running difference images before and during the first eruption toward the southern edge of the AR. The images are taken from the included STEREO B movie, called Movie 1.

Hinode/EIS covered the upflow regions on both sides of the AR extensively. On the east side, where the magnetic field is dispersed, two distinct upflow areas are visible (AR 10953 $(\mathrm{N})$ and $(\mathrm{S})$ in Table 3). The stationary flow model results for the FP southern region are displayed in the bottom panels of Figure 2. Once again, there is an apparent flow evolution, as is evident from the black line of the model fit (left panel). The derived mean velocity is $15.0 \pm 0.3 \mathrm{~km} \mathrm{~s}^{-1}$, similar to values of $V_{\|}$found for AR 10961. Variations of the velocities around the fitted values are comparable to those in AR 11589, with $\sigma_{t}=3.7 \mathrm{~km} \mathrm{~s}^{-1}$ and $\left\langle\sigma_{\text {obs }}\right\rangle=1.6 \mathrm{~km} \mathrm{~s}^{-1}$, while the values relative to the mean velocity are a factor two higher than in the two previous ARs. High velocities that significantly deviate from the fit are observed when the AR is at approximately $0.1-0.3$ in $X$ after CMP (indicated by the arrows in Figures $2 \mathrm{~g}$ and $2 \mathrm{~h}$ ). This position corresponds to the two times when filaments erupted from the AR. Both eruptions appear to occur in the vicinity of the southern (FP) upflow region close to the EIS raster times. The effect of the filament eruptions is clearly indicated by the significant deviation of the velocity data points from the steady-flow model fit in Figure $2 \mathrm{~g}$ and in the increase in variation around the fitted values up to $15 \mathrm{~km} \mathrm{~s}^{-1}$ in Figure $2 \mathrm{~h}$. The deviation from the model fit is evidence of intrinsic flow evolution in AR 10953. Notwithstanding the filament eruptions, upflows are very stable during the AR limb-to-limb transit.

\subsection{General Results}

We have shown the model fits for three examples in order to demonstrate the various conditions under which upflows persist in ARs. These results are general to the ARs in our study 


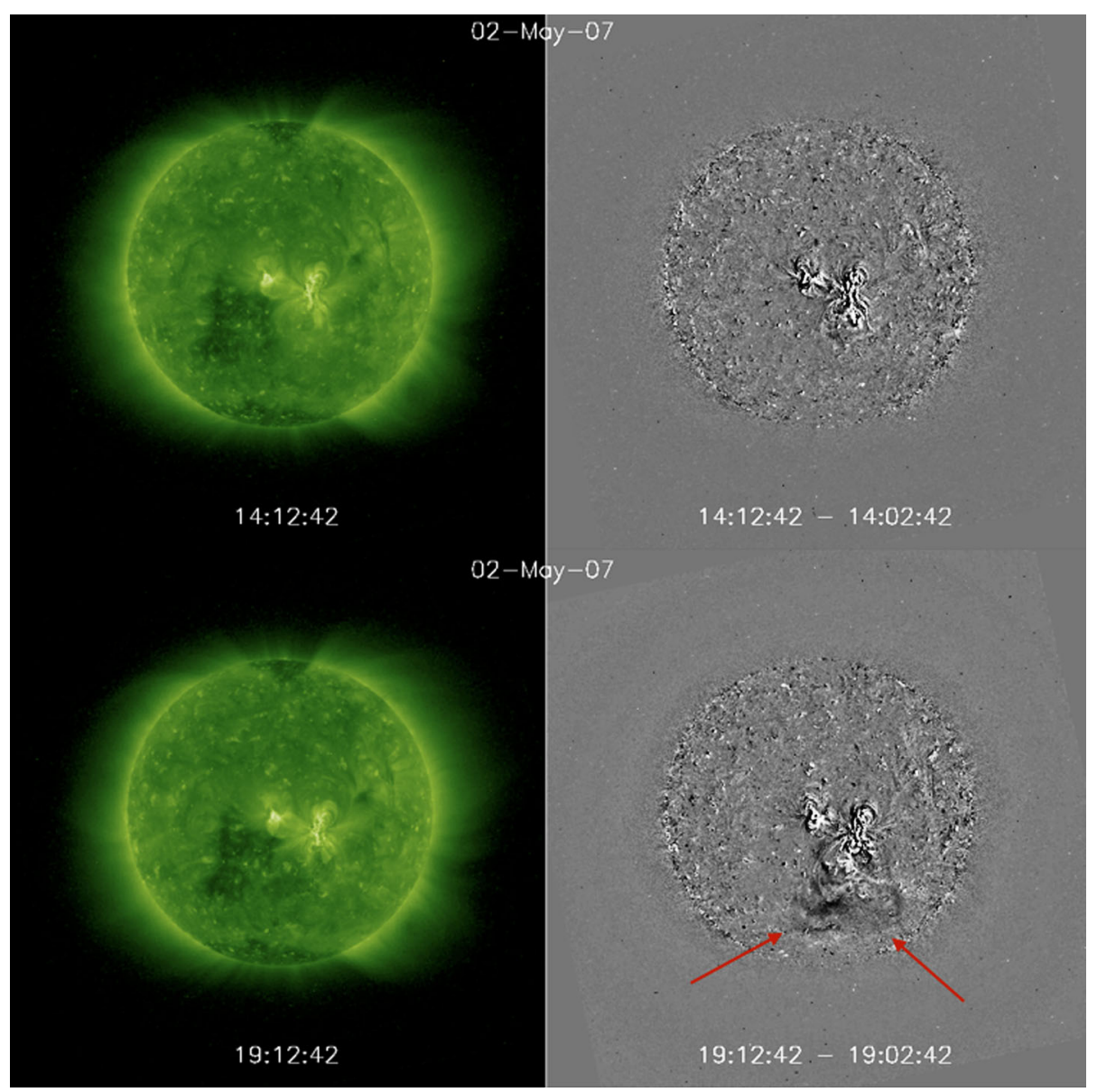

Figure 3 STEREO B $195 \AA$ intensity images (left panels) and running difference images (right panels) of AR 10953 on 2 May 2007. The top and bottom panels show the AR before and during the first CME eruption. Red arrows indicate the front edge of the CME in the running difference image at $\approx 19: 00$ UT. The images are extracted from Movie 1.

since all of them have stationary flows over the LPs and FPs that are observed by Hinode/EIS for 8-9 days during disk transit. Stationary flows are likely to persist for much longer as the flows are seen in ARs with minimum ages that exceed the transit time. This is the case for half of the ARs in our study (see Table 1 for AR minimum ages).

Derived mean velocities are in the range $[9,41] \mathrm{km} \mathrm{s}^{-1}$, and where both polarities are observed by Hinode/EIS, the velocities are similar (see Table 3). Overall, the model fits are quite robust, with the fluctuation of velocities around the fitted values typically within $\pm 1 / 10$ of the mean velocity, except when significant activity is present. Intrinsic stationary flow evolution due to activity such as flux emergence, flaring, and eruptions is indicated by deviations from the model fits, as is shown for AR 10953 (Figures $2 \mathrm{~g}$ and $2 \mathrm{~h}$ ). Such periods of activity are easily identified from the residual velocities to the fit and are removed from the data set. Then the fit is performed again to derive the velocity parameters and the standard deviations outside the activity periods (Table 3 ). 
The derived inclination angles of upflows for all ARs are given in Table 3. AR upflows typically fan away from the AR core by $40^{\circ}$ to near vertical $\left(4^{\circ}\right)$ for the dispersed FPs. The spread of inclination angles is more extensive for LPs with flows angled from $29^{\circ}$ inclined toward to $28^{\circ}$ directed away from the AR. However, with the exception of AR 10926 and AR 10961 (one of the two LP upflows), values of $\delta$ are consistent with those of the FPs, i.e. flows are tilted away from the AR.

\section{Line Widths}

A stationary flow model analysis of the Fe XII spectral line width $[W]$ was performed by fitting the data with

$$
W=W_{0}+W_{r}^{\prime} \sqrt{1-X^{2}-Y^{2}}-W_{\varphi} \frac{X}{1-Y^{2}},
$$

where $X$ is the east-west normalized AR position, $W_{0}$ is a constant that includes all width contributions independent of position, $W_{r}^{\prime}$ takes the flow dispersion in radial and latitudinal directions into account, similar to $V_{r}^{\prime}$ in Equation (2), and $W_{\varphi}$ does the same in the longitudinal direction (see Section 4.4 in Démoulin et al., 2013). Line widths free of instrumental broadening are given as FWHM in $\AA$.

Model fits for two extreme cases are shown in Figure 4. AR 11389 FP (Figures 4b and 4d) exhibits a relatively strong dependence of line width evolution on $X$, as is the case with LOS
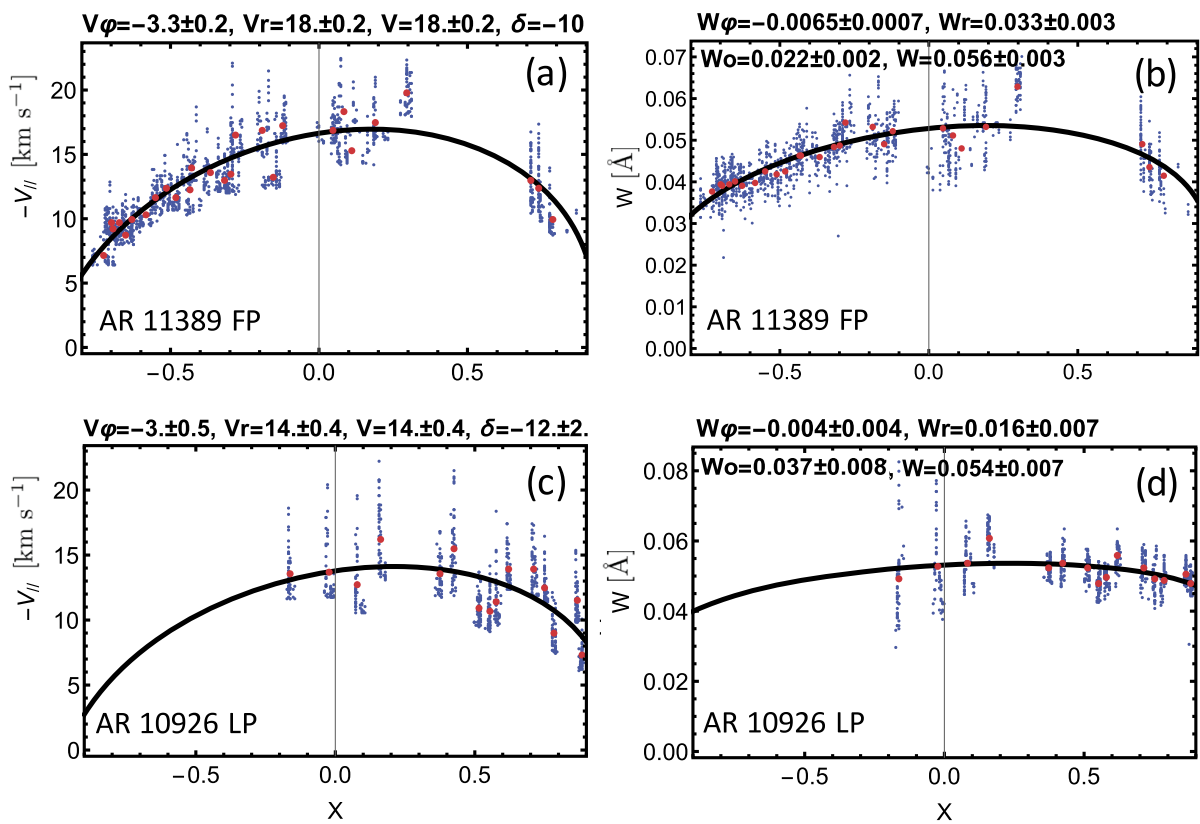

Figure 4 Left panels: Steady-flow model results for AR 11389 FP and AR 10926 LP showing the dependence of the LOS upflow velocity $\left[V_{\|}\right]$on $X$ (east-west normalized AR position). Right panels: Model results for line widths of AR 11389 FP and AR 10926 LP. See the caption to Figure 2 for the definition of the variables and the description of the plots. AR 11389 FP shows strong rotational effects on both LOS velocity and line width, whereas AR 10926 LP presents weak effects for line width. 
upflow velocity. Little activity was evident in this AR after its first few days on the disk (Baker et al., 2015).

Conversely, AR 10926 LP has a weak dependence of line width on $X$ (Figure 4d) compared to the dependence present in $V_{\|}$(Figure 4c). Significant and persistent flux emergence occurs when the AR is in the western hemisphere, thus introducing coronal perturbations that dominate the rotational effects in $W$.

The quantity $\left(W_{r}^{\prime 2}+W_{\varphi}^{2}\right)^{1 / 2} / W_{0}$ represents the relative effect of the rotation over $W_{0}$, which is the contribution to line width not affected by solar rotation. Columns 4 and 5 of Table 3 give values for the FP and the LP of all ARs where available. AR polarities with values approaching or exceeding unity have relatively strong rotational effects on line widths. In such cases, these effects can be detected above $W_{0}$, which includes thermal width (0.022 $\AA$ for Fe XII) and intrinsic fluctuations. Occasionally, the variations of $W$ are falsely interpreted by the fit, e.g. for AR $10942 \mathrm{FP}$, which presents an upward-curved fit. For another example, AR $11575(\mathrm{~N}) \mathrm{FP}$, the fits yield a non-physical result because $W_{0}<0$. This may occur when significant activity is present.

In general, the effect of rotation on $W$ appears to be weaker than on $V_{\|}$. A case in point is AR 11564, which has strong apparent velocities of $30 \mathrm{~km} \mathrm{~s}^{-1}$ for both polarities; however, the rotational effects are marginal for the FP, while they are strong for the LP. Following the proposal of Démoulin et al. (2013), the sensitivity of $W$ to rotational effects is expected to be due to the Doppler velocity differences within the same Hinode/EIS pixel. The distribution of the velocities increases the observed line width. For collimated flows, this effect also has a positional dependence due to the projection along the line of sight. For many of the ARs in our study, the differential effect is weaker than the mean effect of the global line shift (due to the mean plasma velocity), and as a consequence may not be large enough to be visible above $W_{0}$. This implies that rotational effects can be masked easily even without any activity. The addition of activity increases $W_{0}$ and transforms $W_{r}^{\prime}$ and $W_{\varphi}$, which additionally masks the effect of solar rotation. Finally, as is the case with $V$, we find no relation between the values of $W$ and the age of the ARs (cf. Table 1 to Table 3).

\section{Comparison of Deduced Inclinations and LFFF Extrapolation of AR 10926}

We compute the magnetic field topology of AR 10926 during its disk transit on 30 November 2006 at 16:25 UT, 1 December at 16:28 UT, and 3 December at 15:32 UT. The LOS magnetic field is extrapolated to the corona using a constant $\alpha$ linear force-free field (LFFF) configuration where $\boldsymbol{J} \times \boldsymbol{B}=0$ and $\nabla \times \boldsymbol{B}=\alpha \boldsymbol{B}$ (Démoulin et al., 1997). The left panel of Figure 5 shows the LFFF model results for 1 December when the AR was close to CMP. Similar results are found at other times. As in Démoulin et al. (2013), the inclination deduced from the stationary-flow model is compared with that of the LFFF extrapolation. Field lines rooted in the strong upflow region of the FP form two groups, one of which connects to the LP within the AR, and the other group connects outside of the computational box (pink and black field lines, respectively, in Figure 5). The ranges of inclination angles are $\left[-40^{\circ},-10^{\circ}\right]$ for the pink and $\left[-60^{\circ},-40^{\circ}\right]$ for the black field lines. Unusually, compared to other ARs, inclination angles of the LP are within the range $\left[-20^{\circ}, 10^{\circ}\right]$ with a number of field lines inclined to the east, i.e. toward the center of the AR. 

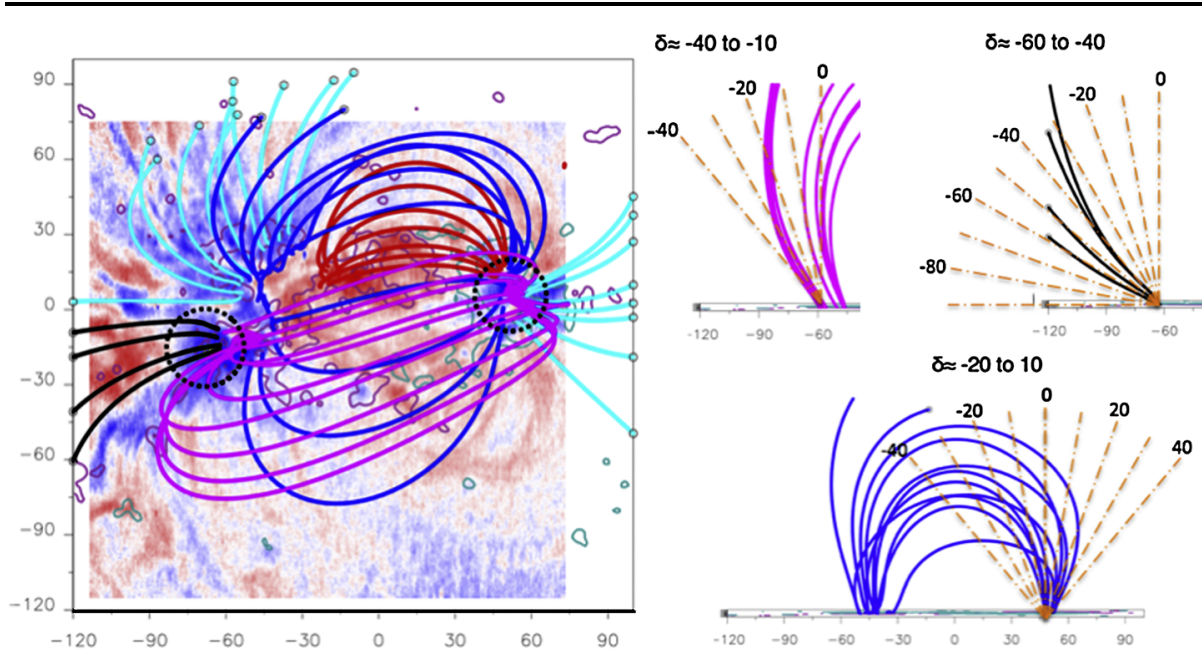

Figure 5 Left panel: Linear force-free field extrapolation for AR 10926 overlaid on Hinode/EIS Doppler velocity map at 16:28 UT on 1 December 2006. Dashed circles indicate regions of strong upflows for FP and LP. Right panels: Inclinations of field lines rooted at locations of strong upflows for FP (top) and LP (bottom).

The deduced inclinations $[\delta]$ from EIS velocities reflect the asymmetry of the opposite polarities in the extrapolation and coronal observations with inclinations for FP of $\delta=-40 \pm 1^{\circ}$ and for LP of $\delta=-12 \pm 2^{\circ}$, i.e. both inclined toward the east. Qualitatively, the steady-flow model and extrapolation inclination angles are broadly comparable, but there is a wide range of inclinations in the extrapolations.

Sources of discrepancies between EIS upflow and magnetic extrapolation deduced directions may be due to a number of factors:

i) We derive the mean inclination of the strongest flows over many days compared to a single snapshot of a model representing all AR field line populations.

ii) The photospheric footpoints of the flows are difficult to estimate so that the correspondence with the computed QSLs and the selected field lines is approximate.

iii) Activity such as the flux emergence observed in this AR can influence the derived parameters of the steady-flow model.

iv) LFFF extrapolations are computed assuming constant $\alpha$, but the AR field may have varying $\alpha$ values as it evolves during disk transit. This is the case for AR 10926 (extrapolations not shown here).

The above sources of discrepancies between EIS upflow directions and computed field lines are present for all ARs, with the only differences in the relative importance of the above factors. Globally, we can only expect a broad coherence between the derived inclinations from the two data sources.

\section{Upflow Pairs - AR 11575}

Three ARs in our sample have more than a single pair of upflow streams that are fitted with the stationary flow model. The flows in AR 10953 can be divided into northern and southern 


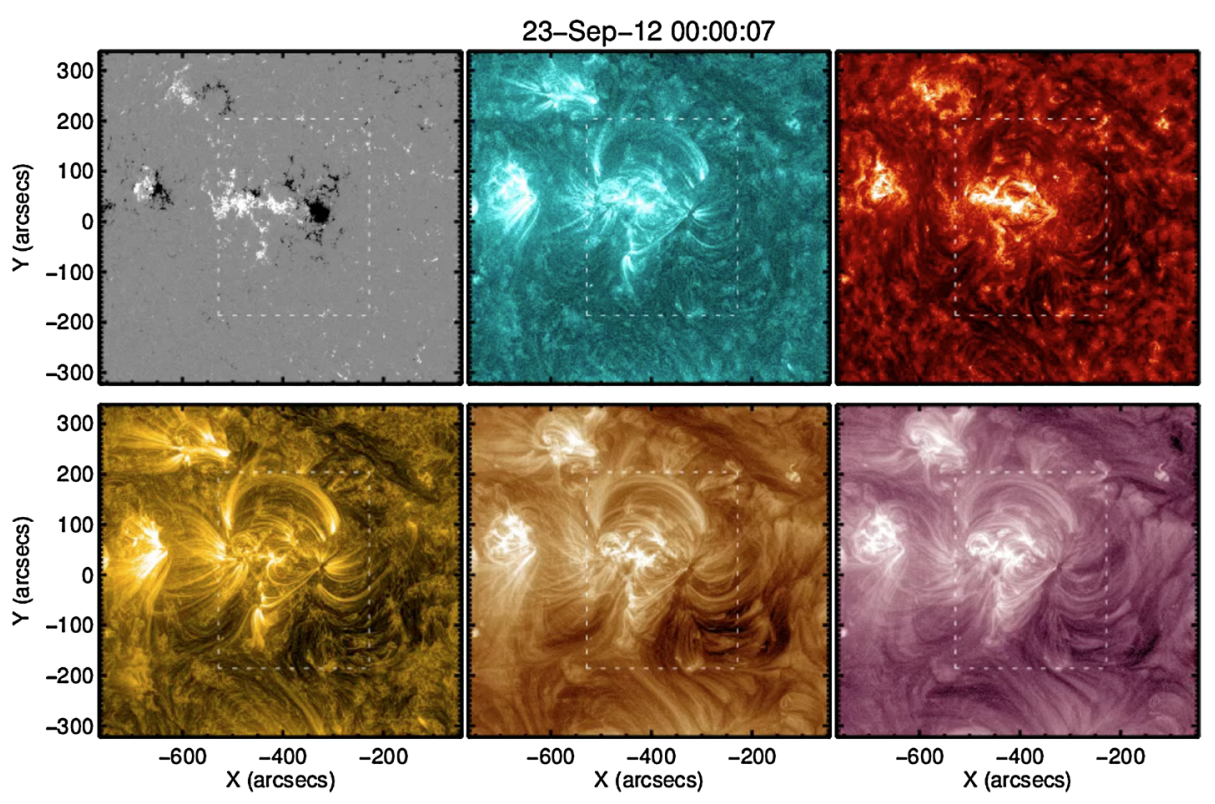

Figure 6 Clockwise from upper left: SDO/HMI magnetogram, SDO/AIA $131 \AA$, $304 \AA$, $211 \AA$, $193 \AA$, and $171 \AA$ images of AR 11575. Hinode/EIS FOV is indicated by dashed white boxes. The image is extracted from Movie 2.

streams. AR 10961 has two upflow regions on the western side. AR 11575 has four distinct flow pairs, one each in the northern, central, and southern areas within the AR, and one to the far south, which is present only during the later EIS observations. Model results are provided in Table 3.

Here we focus on AR 11575, which is featured in Figure 6 and Movie 2, which are both composed of observations from the Heliospheric Magnetic Imager (HMI) and Atmospheric Imaging Assembly (AIA) onboard the Solar Dynamics Observatory (SDO). The AR is complex, formed by several bipoles that are evident in the HMI magnetograms. In addition, loops rooted in the positive FP are connected to both the AR LP and to the negative polarity of the nearby AR 11577 located on the eastern side of AR 11575 (see Movie 2). The strong connectivity between the ARs affects the eastern upflows, which differ from the typical flow regions observed in the isolated simple bipolar ARs in our sample. Parts of the upflows are located at the border of the extended FP rather than directly above the FP.

Individual flow pairs are identified in the Doppler velocity maps and are indicated by boxes in Figure 7 showing Hinode/EIS Fe XII intensity and Doppler velocity maps with corresponding SDO/HMI magnetograms for 23 and 28 September. On 23 September, there are three flow pairs, and on 28 September, additional upflow streams appear together to the far south of the AR. Upflows occur over pairs of opposite-polarity magnetic field patches. To the north, upflows occur at the base of long regions that are close to potential loops that connect to the dispersed magnetic field. Short compact loops within the AR core connect the intact leading spot with the FP. To the south, the upflows occur in sheared loops that connect to very weak dispersed field. 

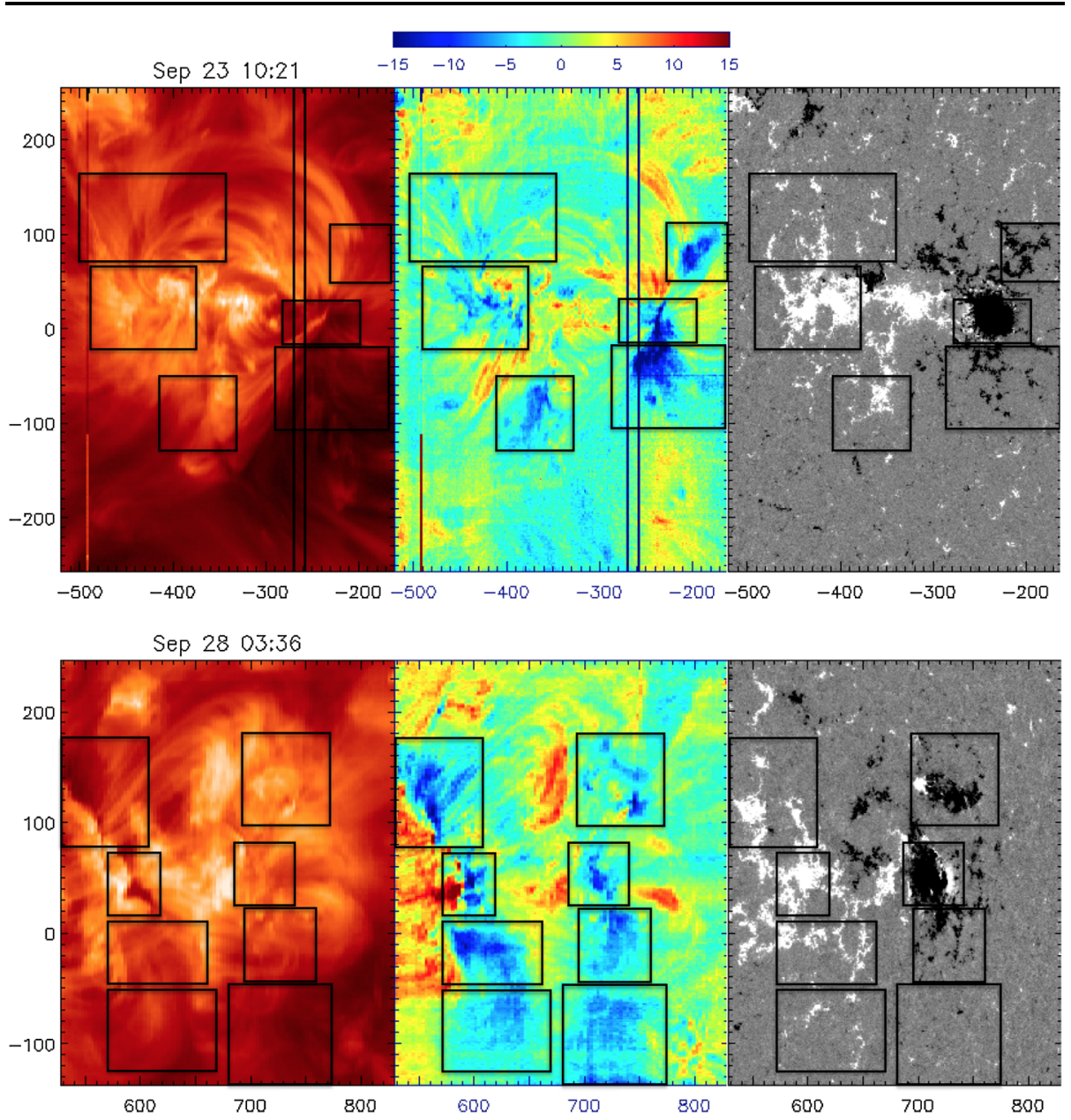

Figure 7 EIS Fe XII intensity (left), Doppler velocity maps (center), and SDO/HMI magnetogram (right) of AR 11575 on 23 (top) and 28 (bottom) September 2012. Boxes indicate upflow pairs corresponding to different magnetic bipoles. Axes have units of arcsecs. The color bar has units of $\mathrm{km} \mathrm{s}^{-1}$.

We are able to fit the model to the three pairs observed on 23 September, but there are too few data to fit the southernmost pair. Derived model velocities are highly comparable within these upflows with [FP, LP] velocities in $\mathrm{km} \mathrm{s}^{-1}$ as follows: $V=[12,12]$ for the northern pair, $V=[12,16]$ for the central pair, and $V=[11,23]$ for the southern pair. The derived $V$ values for the northern and southern pairs are likely to be underevaluated because we do not have measurements of the components of $V$ in the north-south direction. Moreover, the model inclinations may not be well estimated because only relatively few rasters are available on the eastern side before CMP. The near vertical inclination angle of the FP upflow of the southern pair (see Table 3 ) is probably the result of a combination of the connectivity with AR 11577 and the low data coverage where $X<0$. 
Despite the complex configuration of AR 11575, its flow pairs exhibit strong apparent evolution comparable to that of AR 11589 and AR 10961 shown in Figure 2. This is also the case of the separate flows and flow pairs identified in AR 10953 and AR 10961. For six of the ten ARs where both polarities are observed, the mean velocities derived from the model fits for each associated LP and FP are remarkably similar (see Section 4.3 and Table 3).

Our results indicate that the origin of the upflow pairs is related. It is unlikely that consistently similar flow velocities occurring on both sides of the ARs would be maintained for timescales of days and weeks unless the stationary flows are driven by a large-scale global mechanism rather than a local mechanism that acts at a single polarity. The scenario of AR upflows originating from reconnection along QSLs between overpressure AR loops and nearby underpressure loops is consistent with such a mechanism because QSLs are defined by the global properties of the magnetic configuration of the AR and its surroundings (Démoulin, 2007). Moreover, Mandrini et al. (2015) showed that the upflows and QSLs evolve in parallel, both temporarily and spatially.

\section{Summary and Conclusions}

The aim of this study is to use Hinode/EIS data to constrain the geometry and nature of large-scale upflows that are present on both sides of ARs. We use the simple stationary-flow model applied to AR 10978 in Démoulin et al. (2013) to deduce the mean velocity [V], mean line width $[W]$, and inclination angle $[\delta]$ for nine ARs with reasonable limb-to-limb EIS coverage. By separating and characterizing apparent from intrinsic upflow evolution, we set constraints on the mechanisms that are able to drive these large-scale persistent upflows. Our main results are listed below.

- All ARs in our study have stationary flows. As a consequence, the observed long-term evolution of the collimated large-scale and persistent flows is largely due to the evolution of the LOS projection during disk transit.

- In most cases, derived velocities $[V]$ for the following and leading polarities are similar. This holds for asymmetric ARs (e.g. AR 10926 and AR 10961), indicating that the magnetic field strength does not drive the stationary flows, i.e. there is no direct magnetic acceleration as there is with CMEs.

- In general, ARs with stronger flows, i.e. higher mean line-of-sight velocities, show stronger rotational effects.

- Intrinsic flow evolution that is due to activity, such as flux emergence, jets, flaring, and CMEs, appears as a deviation from the model fit (e.g. AR 10953, Figures 2g and 2h). However, since such phenomena have different timescales, ranging from few tens of minutes (flares) to days (emergence), they affect the observed velocity differently. They also affect the estimated steady flow with different amplitudes, and CMEs have the strongest effect. However, because of the different timescales, they can be identified in the velocity data and removed to derive the upflow properties.

- Our study demonstrates that there is no evidence of a relationship between flow evolution and AR age, which is consistent with the results of Zangrilli and Poletto (2016), who found with SOHO/UVCS that upflows remain for the entire lifetime of an AR.

- The effect of solar rotation on line width $[W]$ is clearly present, but weaker than its effect on $V$ for the nine new ARs in our study. This contrasts with the strong dependence of both $V$ and $W$ on AR position in the case of AR10978 (Doschek et al., 2008; Bryans, Young, and Doschek, 2010; Démoulin et al., 2013). The line-broadening dependence 
on AR position is probably due to a large dispersion of velocities along the main flow direction. This effect is weaker than the global line shift, so that thermal width and activity can easily mask the rotational effects on $W$.

- For the set of studied ARs, the following polarity $\delta=\left[0^{\circ}, 40^{\circ}\right]$ is tilted away from the AR core. The leading polarity inclinations have a greater spread with $\delta=\left[-33^{\circ}, 28^{\circ}\right]$ such that the leading-polarity upflows tilt both toward and away from the center of the AR.

- Deduced inclination angles [ $\delta]$ for both polarities of AR 10926 are broadly comparable to those obtained from an LFFF extrapolation of the AR. The $\delta$ results of the stationary-flow model reflect the asymmetric topology of the AR that is evident in the extrapolation and coronal observations.

- Independent flow pairs identified in three more complex ARs display apparent evolution, similar to the simple, isolated bipolar ARs in the sample. The upflows appear in pairs with similar velocities in the following and leading AR polarities, which indicates that the same process is taking place at both polarities of the same pair.

Blueshifted upflows are a common feature of ARs observed by Hinode/EIS throughout its ten-year mission. Our results imply that the stationary component of upflows occurring on either side of ARs are in fact related. Moreover, stationary flows occur in pairs whether the ARs are isolated bipoles common at solar minimum or more complex interconnected multipolar regions observed during solar maximum. This result constrains the possible upflowdriving mechanisms. Mechanisms acting locally at one polarity, e.g. waves, nanoflares, or jets, are unlikely to produce globally stationary upflows with the same characteristics unless they are synchronized by another mechanism such as magnetic reconnection. Our results are in agreement with a model where reconnection occurs along quasi-separatrix layers (QSLs) between overpressure AR loops and neighboring underpressure loops (Baker et al., 2009; Bradshaw, Aulanier, and Del Zanna, 2011; Del Zanna et al., 2011; van Driel-Gesztelyi et al., 2012; Démoulin et al., 2013; Mandrini et al., 2015).

Acknowledgements The authors would like to thank Lidia van Driel-Gesztelyi for fruitful discussions in preparing this manuscript and David Long for making the movies. We thank the anonymous referee for their constructive and informative comments. Hinode is a Japanese mission developed and launched by ISAS/JAXA, collaborating with NAOJ as a domestic partner, NASA and STFC (UK) as international partners. Scientific operation of Hinode is by the Hinode science team organized at ISAS/JAXA. This team mainly consists of scientists from institutes in the partner countries. Support for the post-launch operation is provided by JAXA and NAOJ (Japan), STFC (UK), NASA, ESA, and NSC (Norway). DB is funded under STFC consolidated grant number ST/N000722/1. CHM acknowledges financial support from grants PICT 2012-0973 (ANPCyT), PIP 2012-01-403 (CONICET), and UBACyT 20020130100321. CHM is a member of the Carrera del Investigador Científico (CONICET).

Disclosure of Potential Conflicts of Interest The authors declare that they have no conflicts of interest.

Open Access This article is distributed under the terms of the Creative Commons Attribution 4.0 International License (http://creativecommons.org/licenses/by/4.0/), which permits unrestricted use, distribution, and reproduction in any medium, provided you give appropriate credit to the original author(s) and the source, provide a link to the Creative Commons license, and indicate if changes were made.

\section{Appendix}

Table 4 gives a brief description of the EIS studies for the different active regions presented in the article. 
Table 4 Hinode/EIS study details for the data used in this work. Sparse raster is a scanning raster where the step size exceeds the slit width.

\begin{tabular}{|c|c|c|c|c|c|}
\hline Study No. & $\begin{array}{l}\text { FOV } \\
(\operatorname{arcsec})\end{array}$ & $\begin{array}{l}\text { Exposure time } \\
\text { (seconds) }\end{array}$ & $\begin{array}{l}\text { Slit } \\
(\operatorname{arcsec})\end{array}$ & $\begin{array}{l}\text { Total raster time } \\
\text { (hours) }\end{array}$ & Comments \\
\hline 3 & $256 \times 512$ & 60 & 1 & 4.3 & \\
\hline 5 & $256 \times 256$ & 30 & 1 & 2.1 & \\
\hline 7 & $512 \times 256$ & 30 & 1 & 4.3 & \\
\hline 9 & $256 \times 256$ & 10 & 1 & 0.7 & \\
\hline 10 & $256 \times 256$ & 40 & 1 & 2.8 & \\
\hline 36 & $240 \times 240$ & 5 & 1 & 0.3 & \\
\hline 37 & $240 \times 240$ & 5 & 2 & 0.2 & \\
\hline 45 & $128 \times 372$ & 60 & 1 & 2.1 & \\
\hline 46 & $256 \times 256$ & 15 & 1 & 1.1 & \\
\hline 50 & $128 \times 128$ & 25 & 1 & 0.9 & \\
\hline 84 & $120 \times 256$ & 20 & 1 & 0.7 & \\
\hline 85 & $256 \times 256$ & 10 & 1 & 0.7 & \\
\hline 95 & $257 \times 408$ & 30 & 1 & 2.1 & \\
\hline 174 & $82 \times 400$ & 25 & 2 & 0.3 & \\
\hline 176 & $82 \times 400$ & 25 & 2 & 0.3 & \\
\hline 179 & $100 \times 240$ & 25 & 2 & 0.3 & \\
\hline 180 & $180 \times 512$ & 50 & 2 & 0.8 & Sparse \\
\hline 261 & $360 \times 512$ & 70 & 2 & 3.5 & \\
\hline 361 & $200 \times 400$ & 10 & 2 & 0.3 & \\
\hline 381 & $195 \times 280$ & 45 & 2 & 0.8 & Sparse \\
\hline 437 & $240 \times 512$ & 60 & 1 & 2.0 & Sparse \\
\hline 454 & $303 \times 384$ & 40 & 2 & 1.1 & Sparse \\
\hline 471 & $360 \times 512$ & 60 & 1 & 3.0 & Sparse \\
\hline 480 & $480 \times 512$ & 15 & 1 & 1.0 & Sparse \\
\hline
\end{tabular}

\section{References}

Aulanier, G., Pariat, E., Démoulin, P., DeVore, C.R.: 2006, Slip-running reconnection in quasi-separatrix layers. Solar Phys. 238, 347. DOI. ADS.

Baker, D., van Driel-Gesztelyi, L., Mandrini, C.H., Démoulin, P., Murray, M.J.: 2009, Magnetic reconnection along quasi-separatrix layers as a driver of ubiquitous active region outflows. Astrophys. J. 705, 926. DOI. ADS.

Baker, D., Brooks, D.H., Démoulin, P., Yardley, S.L., van Driel-Gesztelyi, L., Long, D.M., Green, L.M.: 2015, FIP bias evolution in a decaying active region. Astrophys. J. 802, 104. DOI. ADS.

Bradshaw, S.J., Aulanier, G., Del Zanna, G.: 2011, A reconnection-driven rarefaction wave model for coronal outflows. Astrophys. J. 743, 66. DOI. ADS.

Brooks, D.H., Warren, H.P.: 2012, The coronal source of extreme-ultraviolet line profile asymmetries in solar active region outflows. Astrophys. J. Lett. 760, L5. DOI. ADS.

Bryans, P., Young, P.R., Doschek, G.A.: 2010, Multiple component outflows in an active region observed with the EUV imaging spectrometer on hinode. Astrophys. J. 715, 1012. DOI. ADS.

Culhane, J.L., Harra, L.K., James, A.M., Al-Janabi, K., Bradley, L.J., Chaudry, R.A., Rees, K., Tandy, J.A., Thomas, P., Whillock, M.C.R., Winter, B., Doschek, G.A., Korendyke, C.M., Brown, C.M., Myers, S., Mariska, J., Seely, J., Lang, J., Kent, B.J., Shaughnessy, B.M., Young, P.R., Simnett, G.M., Castelli, C.M., Mahmoud, S., Mapson-Menard, H., Probyn, B.J., Thomas, R.J., Davila, J., Dere, K., Windt, D., Shea, J., Hagood, R., Moye, R., Hara, H., Watanabe, T., Matsuzaki, K., Kosugi, T., Hansteen, V., Wikstol, Ø.: 2007, The EUV imaging spectrometer for hinode. Solar Phys. 243, 19. DOI. ADS. 
Culhane, J.L., Brooks, D.H., van Driel-Gesztelyi, L., Démoulin, P., Baker, D., DeRosa, M.L., Mandrini, C.H., Zhao, L., Zurbuchen, T.H.: 2014, Tracking solar active region outflow plasma from its source to the near-earth environment. Solar Phys. 289, 3799. DOI. ADS.

De Pontieu, B., McIntosh, S.W.: 2010, Quasi-periodic propagating signals in the solar corona: The signature of magnetoacoustic waves or high-velocity upflows? Astrophys. J. 722, 1013. DOI. ADS.

De Pontieu, B., McIntosh, S.W., Hansteen, V.H., Schrijver, C.J.: 2009, Observing the roots of solar coronal heating in the chromosphere. Astrophys. J. Lett. 701, L1. DOI. ADS.

Del Zanna, G.: 2008, Flows in active region loops observed by hinode EIS. Astron. Astrophys. 481, L49. DOI. ADS.

Del Zanna, G., Aulanier, G., Klein, K.-L., Török, T.: 2011, A single picture for solar coronal outflows and radio noise storms. Astron. Astrophys. 526, A137. DOI. ADS.

Démoulin, P.: 2007, Where will efficient energy release occur in 3-D magnetic configurations? Adv. Space Res. 39, 1367. DOI. ADS.

Démoulin, P., Hénoux, J.C., Priest, E.R., Mandrini, C.H.: 1996, Quasi-separatrix layers in solar flares. I. Method. Astron. Astrophys. 308, 643. ADS.

Démoulin, P., Bagala, L.G., Mandrini, C.H., Hénoux, J.C., Rovira, M.G.: 1997, Quasi-separatrix layers in solar flares. II. Observed magnetic configurations. Astron. Astrophys. 325, 305. ADS.

Démoulin, P., Baker, D., Mandrini, C.H., van Driel-Gesztelyi, L.: 2013, The 3D geometry of active region upflows deduced from their limb-to-limb evolution. Solar Phys. 283, 341. DOI. ADS.

Doschek, G.A., Warren, H.P., Mariska, J.T., Muglach, K., Culhane, J.L., Hara, H., Watanabe, T.: 2008, Flows and nonthermal velocities in solar active regions observed with the EUV imaging spectrometer on hinode: A tracer of active region sources of heliospheric magnetic fields? Astrophys. J. 686, 1362. DOI. ADS.

Galsgaard, K., Madjarska, M.S., Vanninathan, K., Huang, Z., Presmann, M.: 2015, Active region upflows. II. Data driven magnetohydrodynamic modelling. Astron. Astrophys. 584, A39. DOI. ADS.

Hara, H., Watanabe, T., Harra, L.K., Culhane, J.L., Young, P.R., Mariska, J.T., Doschek, G.A.: 2008, Coronal plasma motions near footpoints of active region loops revealed from spectroscopic observations with hinode EIS. Astrophys. J. Lett. 678, L67. DOI. ADS.

Harra, L.K., Sakao, T., Mandrini, C.H., Hara, H., Imada, S., Young, P.R., van Driel-Gesztelyi, L., Baker, D.: 2008, Outflows at the edges of active regions: Contribution to solar wind formation? Astrophys. J. Lett. 676, L147. DOI. ADS.

Janvier, M.: 2017, Three-dimensional magnetic reconnection and its application to solar flares. J. Plasma Phys. DOI.

Mandrini, C.H., Baker, D., Démoulin, P., Cristiani, G.D., van Driel-Gesztelyi, L., Vargas Domínguez, S., Nuevo, F.A., Vásquez, A.M., Pick, M.: 2015, Parallel evolution of quasi-separatrix layers and active region upflows. Astrophys. J. 809, 73. DOI. ADS.

Marsch, E., Tian, H., Sun, J., Curdt, W., Wiegelmann, T.: 2008, Plasma flows guided by strong magnetic fields in the solar corona. Astrophys. J. 685, 1262. DOI. ADS.

McIntosh, S.W., De Pontieu, B.: 2009, High-speed transition region and coronal upflows in the quiet sun. Astrophys. J. 707, 524. DOI. ADS.

Murray, M.J., Baker, D., van Driel-Gesztelyi, L., Sun, J.: 2010, Outflows at the edges of an active region in a coronal hole: A signature of active region expansion? Solar Phys. 261, 253. DOI. ADS.

Ofman, L., Wang, T.J., Davila, J.M.: 2012, Slow magnetosonic waves and fast flows in active region loops. Astrophys. J. 754, 111. DOI. ADS.

Okamoto, T.J., Tsuneta, S., Lites, B.W., Kubo, M., Yokoyama, T., Berger, T.E., Ichimoto, K., Katsukawa, Y., Nagata, S., Shibata, K., Shimizu, T., Shine, R.A., Suematsu, Y., Tarbell, T.D., Title, A.M.: 2008, Emergence of a helical flux rope under an active region prominence. Astrophys. J. Lett. 673, L215. DOI. ADS.

Peter, H.: 2010, Asymmetries of solar coronal extreme ultraviolet emission lines. Astron. Astrophys. 521, A51. DOI. ADS.

Sakao, T., Kano, R., Narukage, N., Kotoku, J., Bando, T., DeLuca, E.E., Lundquist, L.L., Tsuneta, S., Harra, L.K., Katsukawa, Y., Kubo, M., Hara, H., Matsuzaki, K., Shimojo, M., Bookbinder, J.A., Golub, L., Korreck, K.E., Su, Y., Shibasaki, K., Shimizu, T., Nakatani, I.: 2007, Continuous plasma outflows from the edge of a solar active region as a possible source of solar wind. Science 318, 1585. DOI. ADS.

Su, Y., van Ballegooijen, A., Lites, B.W., Deluca, E.E., Golub, L., Grigis, P.C., Huang, G., Ji, H.: 2009, Observations and nonlinear force-free field modeling of active region 10953. Astrophys. J. 691, 105. DOI. ADS.

Testa, P., De Pontieu, B., Hansteen, V.: 2016, High spatial resolution Fe XII observations of solar active regions. Astrophys. J. 827, 99. DOI. ADS.

Tian, H., McIntosh, S.W., De Pontieu, B., Martínez-Sykora, J., Sechler, M., Wang, X.: 2011, Two components of the solar coronal emission revealed by extreme-ultraviolet spectroscopic observations. Astrophys. J. 738, 18. DOI. ADS. 
Tian, H., McIntosh, S.W., Wang, T., Ofman, L., De Pontieu, B., Innes, D.E., Peter, H.: 2012, Persistent doppler shift oscillations observed with hinode/EIS in the solar corona: Spectroscopic signatures of Alfvénic waves and recurring upflows. Astrophys. J. 759, 144. DOI. ADS.

van Driel-Gesztelyi, L., Green, L.M.: 2015, Evolution of active regions. Living Rev. Solar Phys. 12, 1. DOI. ADS.

van Driel-Gesztelyi, L., Culhane, J.L., Baker, D., Démoulin, P., Mandrini, C.H., DeRosa, M.L., Rouillard, A.P., Opitz, A., Stenborg, G., Vourlidas, A., Brooks, D.H.: 2012, Magnetic topology of active regions and coronal holes: Implications for coronal outflows and the solar wind. Solar Phys. 281, 237. DOI. ADS.

Vanninathan, K., Madjarska, M.S., Galsgaard, K., Huang, Z., Doyle, J.G.: 2015, Active region upflows. I. Multi-instrument observations. Astron. Astrophys. 584, A38. DOI. ADS.

Verwichte, E., Marsh, M., Foullon, C., Van Doorsselaere, T., De Moortel, I., Hood, A.W., Nakariakov, V.M.: 2010, Periodic spectral line asymmetries in solar coronal structures from slow magnetoacoustic waves. Astrophys. J. Lett. 724, L194. DOI. ADS.

Wang, T.J., Ofman, L., Davila, J.M., Mariska, J.T.: 2009, Hinode/EIS observations of propagating lowfrequency slow magnetoacoustic waves in fan-like coronal loops. Astron. Astrophys. 503, L25. DOI. ADS.

Warren, H.P., Ugarte-Urra, I., Young, P.R., Stenborg, G.: 2011, The temperature dependence of solar active region outflows. Astrophys. J. 727, 58. DOI. ADS.

Young, P.R., Watanabe, T., Hara, H., Mariska, J.T.: 2009, High-precision density measurements in the solar corona. I. Analysis methods and results for Fe XII and Fe XIII. Astron. Astrophys. 495, 587. DOI. ADS.

Zangrilli, L., Poletto, G.: 2016, Evolution of active region outflows throughout an active region lifetime. Astron. Astrophys. 594, A40. DOI. ADS. 